\title{
Transverse intrinsic localized modes in monatomic chain and in graphene
}

\author{
V. Hizhnyakov ${ }^{\text {a }}$, M. Klopov ${ }^{\mathrm{b}}$, A. Shelkan ${ }^{\mathrm{a}, *}$ \\ ${ }^{a}$ Institute of Physics, University of Tartu, Ravila 14c, 50411 Tartu, Estonia \\ ${ }^{\mathrm{b}}$ Department of Physics, Faculty of Science, Tallinn University of Technology, \\ Ehitajate 5, 19086 Tallinn, Estonia
}

\begin{abstract}
In this paper an analytical and numerical study of anharmonic vibrations of monatomic chain and graphene in transverse (perpendicular) with respect to the chain/plane direction is presented. Due to the lack of odd anharmonicities and presence of hard quartic anharmonicity for displacements in this direction, there may exist localized anharmonic transverse modes with the frequencies above the spectrum of the corresponding phonons. Although these frequencies are in resonance with longitudinal (chain) or in-plane (graphene) phonons, the modes can decay only due to a weak anharmonic process. Therefore the lifetime of these vibrations may be very long. E.g. in the chain, according to our theoretical and numerical calculations it may exceed $10^{10}$ periods. We call these vibrations as transverse intrinsic localized modes.
\end{abstract}

Key words: Lattice vibrations; Anharmonicity; Intrinsic localized mode.

\section{Introduction}

It is a well-known fact that point defects in crystals may cause an appearance of spatially localized vibrations, called local modes (see, e.g. [1]). The frequency of such modes lies outside the phonon spectrum, which prevents the spreading of the vibrations to the bulk. Besides, resonant or pseudolocal modes with the frequencies inside the phonon spectrum may also exist. They appear when the frequency of the leading vibration associated with the defect gets to a region of small density of states (DOS) of phonons. Unlike local

\footnotetext{
* Corresponding author.

Email address: shell@ut.ee (A. Shelkan).
} 
modes, which are stable in harmonic approximation, these modes can live only a finite time due to the emission of resonant phonons. However, because of the small DOS of resonant phonons the interaction of the latter with pseudolocal mode is weak, due to what the lifetime of the mode becomes long. A well-known case of long-living pseudolocal modes in 3D lattices is the one by the defect of large mass [1].

Spatially localized modes may exist also in perfect nonlinear lattices. Such modes in chains with cubic and quartic anharmonicity were first described by A. M. Kosevich and A. S. Kovalev [2]. They have found that for sufficiently strong quartic anharmonicity there exist vibrational states localized in the space and periodic in time; the frequency of these vibrations exceeds the maximum frequency of phonons. The authors [2] restricted their consideration with large-size vibrations and the frequency close to the top of the phonon spectrum. Small size localized vibrations in anharmonic lattices were introduced by Dolgov [3] and then by A. J. Sievers and S. Takeno [4] and were called "intrinsic localized modes" (ILMs).

In the numerical studies of ILMs different two-body potentials (Lennard-Jones, Born-Mayer-Coulomb, Toda, and Morse potentials and their combinations) have been used (see, e.g. [5,6,7]). All these potentials have strong odd anharmonicities and show a strong softening with the increasing of the vibrational amplitude. The ILMs found in these simulations always drop down from optical band(s) into the phonon gap, if there is any. In this connection see Ref. [8], where gap ILMs in NaI were calculated without taking into account longrange interactions and Ref. [9, where the calculations of ILMs in NaI were made taking these interactions into account.

Usually in crystal lattices odd anharmonicities are strong and pair potentials show a strong softening with the increase of vibrational amplitude. Therefore, the dropping down of the frequency of ILMs from optical bands is quite common in 3D lattices. Still, as it was shown in Refs. [10,11,12,13], in some crystals odd anharmonicities are reduced due to multiparticle or covalent interactions. Examples have been given with germanium [10], diamond [11], metallic $\mathrm{Ni}$, $\mathrm{Nb}$ [12], iron [13] and copper [11]; in all these crystals ILMs with frequencies above the top of the phonon band were found in numerical simulations.

However, there are systems in which the odd anharmonicities disappear due to symmetry arguments; at the same time, the quartic anharmonicity is non-zero and it is hard (positive). The examples of such systems are given by linear atomic chains and planar atomic structures (e.g. graphene); in these systems odd anharmonicities disappear for vibrations in the transverse (out-of-chain and out-of-plane) direction. Therefore, one can expect that in these systems there can exist transverse anharmonic local modes with the frequencies above the maximum frequency of the corresponding phonons. The latter frequen- 
cies are usually smaller than the maximum frequency of longitudinal/in-plane phonons. These modes fall in resonance with these phonons and can decay. However, unlike pseudolocal modes and like local modes the interaction causing the decay of these modes is anharmonic. Therefore we call them as transverse ILMs (TILMs). The anharmonic interaction of TILMs with small vibrational amplitudes of atoms is very weak. Hence, one can expect that the lifetime of such TILM may be rather long. Below we will consider TILMs in a monatomic chain and in graphene, both analytically and numerically. Our considerations confirm the aforesaid expectation.

\section{Anharmonic chain}

First we consider the anharmonic monatomic chain and examine the vibrations of its atoms in transverse $(y)$ directions. We suppose that the potential energy $U$ of the chain is given by the sum of pair potentials $V\left(R_{n, n^{\prime}}\right)$, where $R_{n, n^{\prime}}$ is the distance between the atoms $n$ and $n^{\prime}$. The latter potentials can be expanded into the series of atomic displacements. Denoting $d=\left(n-n^{\prime}\right) a, x=x_{n}-x_{n^{\prime}}$, $y=y_{n}-y_{n^{\prime}}$, where $a$ is the atom spacing, $x_{n}$ and $y_{n}$ are the longitudinal and transverse displacements of the atom number $n$ from its equilibrium position, we get $R \equiv R_{n, n^{\prime}}=\sqrt{(d+x)^{2}+y^{2}}$. As y appear as $y^{2}$, any power expansion of $\mathrm{R}$ will have only even powers of $y$. The same holds for $U$. This means that $U$ indeed has no odd anharmonic terms. This is a consequence of the symmetry of the chain with respect to the change of the sign of $y$.

Note one more property of the chain: the term in the expansion of $R$, quadratic with respect to $y$, has the same numerical factor as the term linear with respect to $x$. The same holds also for an arbitrary power of $R$. In the equilibrium state all linear terms with respect to the coordinates $x$ in the potential energy $U$ are cancelled. Therefore, all quadratic terms with respect to $y$ are also cancelled, i.e the frequencies of transverse vibrations in the pair potential approximation tend to zero. As a result long-range fluctuations can be created with little energy cost and since they increase the entropy they are favored. This leads to the instability of the chain with respect to small transverse distortions (see in this connection the Mermin-Wagner theorem [14]).

To get the chain stable one needs to stretch it [15]. In this case the atom spacing $a$ is replaced by $a+s$, where $s$ is stretching. Then the terms in $U$ linear with respect to $x$ and $y^{2}$ are not cancelled any more. Therefore, the elastic springs for transverse vibrations are also nonzero and positive. This results in the appearance of transverse phonons with finite, although small for small stretching maximum frequency $\omega_{t m}$. As these phonons do not have any cubic anharmonicity, but have nonzero positive quartic anharmonicity, low-frequency TILMs with the frequency above the spectrum of transverse 
phonons should exist here.

Let us consider the TILM in a monatomic chain with the Morse pair potential

$$
V=D\left(1-e^{\alpha(a-r)}\right)^{2}
$$

Here $D$ is the energy of dissociation, $\alpha$ is the parameter. We are using dimensionless coordinates with the units corresponding to $a=1$ and the value $\alpha=4$ of the Morse pair potentials of atoms in monatomic metals. For this potential only nearest-neighbor interactions are essential and only these potentials will be taken into account here. We also take for the mass units the mass of the atoms of the chain $(M=1)$. The dissociation energy is chosen so that the unit frequency will correspond to the maximum frequency of longitudinal phonons. In this case the potential energy of the stretched lattice is the sum of the following pair potentials (up to a constant term):

$$
V=\left(1-e^{-4(r-1)}\right)^{2} / 128-x\left(1-e^{-4 s}\right) e^{-4 s} / 16
$$

where $r=\sqrt{(x+1+s)^{2}+y^{2}}$. The last term in Eq. (2) accounts for the effect of the stretching force of the chain in $x$ direction - it changes the equilibrium distance of the atoms from $r=1$ to $r=1+s$. Let us expand the potential into the series of $x$ and $y$ and take into account up to the second-order terms with respect to $x$ and forth-order terms with respect to $y$. We get (up to a constant)

$$
V(x, y) \approx \frac{\nu_{1}}{8} x^{2}+\frac{\nu_{2}}{8} s y^{2}+\frac{\nu_{3}}{8} x y^{2}+\frac{\nu_{4}}{32} y^{4}
$$

where $\nu_{i}$ are dependent on stretching $s$ parameters. In the small $s$ limit $\nu_{i} \approx 1$. If $s=0.05$ then $\nu_{1} \approx 0.522, \nu_{2} \approx 0.707, \nu_{3} \approx 0.463, \nu_{4} \approx 0.441$.

The pair potential of the longitudinal vibrations alone is given by the first term in the right-hand side of Eq. (3). Vibrational frequencies of corresponding phonons equal [1]

$$
\omega_{k}=\sqrt{\nu_{1}(1-\cos (k)) / 2} \text {. }
$$

The maximum frequency of longitudinal phonons corresponds to $k=-\pi$ and equals $\omega_{l m}=\sqrt{\nu_{1}}$. The transverse vibrations alone are described by the pair potential

$$
V(0, y)=\frac{\nu_{2} s}{8} y^{2}+\frac{\nu_{4}}{32} y^{4}
$$


In harmonic approximation $\left(\nu_{4}=0\right)$ the frequencies of corresponding phonons are given by Eq. (4) with $\nu_{2} s$ instead on $\nu_{1}$. The positive quartic anharmonicity in Eq. (5) leads to appearance of the anharmonic modes [2] (called here

as TILMs) with the frequencies $\omega_{0}=\omega_{t m} \sqrt{1+\varepsilon^{2} / 4}$ above the maximum frequency of transverse phonons $\omega_{t m}=\sqrt{\nu_{2} s}$ and with the displacements

$$
y_{n}(t) \approx(-1)^{n} A_{0} \cosh ^{-1}(\varepsilon n) \cos \left(\omega_{0} t\right)
$$

Here $A_{0}$ is the amplitude of the central atom,

$$
\varepsilon=\sqrt{3 \nu_{4}} A_{0} / \omega_{t m}
$$

is the reversed size of the TILM (we use the discrete analog of the derived in Ref. [2] equation (47) for the difference in the displacements of two neighbouring atoms $\chi(x))$. These modes interact with longitudinal phonons and, therefore, they decay. To describe this decay we consider the longitudinal vibrations of atoms in the presence of the TILM. Taking into account Eq. (6) we replace $y$ by $y(t)$ in Eq. (3) and get the following pair potential for this motion:

$$
V(x, t) \approx \frac{\nu_{1}}{8} x^{2}+\frac{\nu_{3}}{16} x y^{2}(0)+\frac{\nu_{3}}{16} x y^{2}(0) \cos \left(2 \omega_{0} t\right) .
$$

The first term in the right-hand side of this equation gives the potential energy of the longitudinal vibrations alone in harmonic approximation. The second and the third terms describe the anharmonic interaction of these and transverse vibrations. At that the second term stands for a small local compression, while the third term describes the force with the frequency $2 \omega_{0}$ periodically changing in time. For all longitudinal phonons, except those with the resonant frequency $\omega_{k}=2 \omega_{0}$, this force causes forced vibrations of atoms with the frequency $2 \omega_{0}$. The resonant term causes the increase of the energy of phonons in time. From energy conservation law it follows that this energy comes from the TILM, i.e. the TILM decays.

\section{Decay rate of transverse ILM in chain}

To find the rate of decay we are considering the equation of motion of the longitudinal phonon with the coordinate

$$
x_{k}=N^{-1 / 2} \sum_{n} x_{n} \sin (k n),
$$


where $N \gg 1$ is the number of atoms in the chain, $k=2 \pi m / N$ is the wave number of the phonon, $m=0, \pm 1, \pm 2, \ldots$ (we use periodic boundary conditions), $x_{n}$ is the displacement of the atom $n$ from its equilibrium position. In the case of pair potential (8) this equation reads

$$
\ddot{x}_{k} \cong-\omega_{k}^{2} x_{k}+\frac{\nu_{3}}{16 N} \sum_{n=-\infty}^{\infty} \sin (k n)\left(\left(y_{0, n+1}-y_{0, n}\right)^{2}-\left(y_{0, n}-y_{0, n-1}\right)^{2}\right) \cos \left(2 \omega_{0} t\right),
$$

where $\omega_{k}$ is the frequency of the longitudinal phonon $k$ given by Eq. (4), $y_{0, n} \equiv y_{n}(0)$ is the initial amplitude of the transverse ILM on atom $n$ given by Eq. (6). The first term in the right-hand side of Eq. (10) stands for the harmonic force stemming from the first term in the right-hand side of Eq. (8) and the second term accounts for the anharmonic force stemming from the third term in the right-hand side of this equation (a small compression of the chain stemming from the second term in the right-hand side of Eq. (8) is neglected). The transverse amplitude $\left|y_{0, n}\right|$ slowly changes with $n$. Therefore, $y_{0, n+1}-y_{0, n} \cong 2 y_{0, n}$ and

$$
\left(y_{0, n+1}-y_{0, n}\right)^{2}-\left(y_{0, n}-y_{0, n-1}\right)^{2} \approx 4 \partial y_{0, n}^{2} / \partial n
$$

Inserting Eq. (11) into Eq. (10) and taking into account Eq. (6), we get

$$
\ddot{x}_{k}+\omega_{k}^{2} x_{k} \cong C_{k} N^{-1 / 2} \cos \left(2 \omega_{0} t\right) \text {, }
$$

where

$$
C_{k} \cong \frac{\nu_{3}}{4} A_{0}^{2} \sum_{n=-\infty}^{\infty} \sin (k n) \frac{\partial}{\partial n}\left(\frac{1}{\cosh ^{2}(\varepsilon n)}\right) \cong-\varepsilon \nu_{3} A_{0}^{2} \sum_{n=0}^{\infty} \sin (k n) \frac{\sinh (\varepsilon n)}{\cosh ^{3}(\varepsilon n)}
$$

By using the Green's function of harmonic oscillator $G_{k}(t)=\omega_{k}^{-1} \sin \left(\omega_{k} t\right)$, the solution of Eq. (12) can be presented in the following form:

$$
x_{k}(t)=x_{0, k} \cos \left(\omega_{k} t\right)+x_{k}^{\prime}(t)
$$

where

$$
x_{k}^{\prime}(t)=\frac{C_{k}}{\sqrt{N} \omega_{k}} \int_{0}^{t} \sin \left(\omega_{k}\left(t-t^{\prime}\right)\right) \cos \left(2 \omega_{0} t^{\prime}\right) d t^{\prime} .
$$

The first term in Eq. (14) $\left(\propto x_{0, k}\right)$ stands for free oscillations, while the second term $\left(x_{k}^{\prime}(t)\right)$ describes the excitation of vibrations by the transverse mode. 
The energy of the excited vibrations is given by the sum of the terms $\omega_{k}^{2} x_{k}^{\prime 2}$ averaged over period and summed over all phonons. This gives

$$
E(t)=\frac{1}{N} \sum_{k} C_{k}^{2}\left\langle\int_{0}^{t} d t^{\prime} \sin \left(\omega_{k}\left(t-t^{\prime}\right)\right) \cos \left(2 \omega_{0} t^{\prime}\right) \int_{0}^{t} d t^{\prime \prime} \sin \left(\omega_{k}\left(t-t^{\prime \prime}\right)\right) \cos \left(2 \omega_{0} t^{\prime \prime}\right)\right\rangle
$$

Let us take into account the relations:

$$
\begin{aligned}
& \sin \left(\omega_{k}\left(t-t^{\prime}\right)\right) \sin \left(\omega_{k}\left(t-t^{\prime \prime}\right)\right)=\left(\cos \left(\omega_{k}\left(t^{\prime}-t^{\prime \prime}\right)\right)-\cos \left(\left(\omega_{k}\left(2 t-t^{\prime}-t^{\prime \prime}\right)\right)\right) / 2,\right. \\
& \cos \left(2 \omega_{0} t^{\prime}\right) \cos \left(2 \omega_{0} t^{\prime \prime}\right)=\left(\cos \left(2 \omega_{0}\left(t^{\prime}-t^{\prime \prime}\right)\right)+\cos \left(2 \omega_{0}\left(t^{\prime}+t^{\prime \prime}\right)\right)\right) / 2 .
\end{aligned}
$$

In the case under consideration frequencies of phonons form a continuous spectrum. In this case, due to the summation (integration) over $k$ the terms in Eq. (16) $\propto \cos \left(\omega_{k}\left(t^{\prime}-t^{\prime \prime}\right)\right)$ and $\propto \cos \left(\left(\omega_{k}\left(2 t-t^{\prime}-t^{\prime \prime}\right)\right)\right.$ essentially differ from zero only for finite values of the arguments $t^{\prime}-t^{\prime \prime}$ and $2 t-t^{\prime}-t^{\prime \prime}$ (of the order of unity). At that the term $\propto \cos \left(\left(\omega_{k}\left(2 t-t^{\prime}-t^{\prime \prime}\right)\right)\right)$ essentially differs from zero only for large $t^{\prime}, t^{\prime \prime} \sim t$ when $\cos \left(2 \omega_{0} t^{\prime}\right)$ and $\cos \left(2 \omega_{0} t^{\prime \prime}\right)$ average the contrubution of this term out. Neglecting now unessential for $t^{\prime}, t^{\prime \prime} \sim t>>\omega_{0}^{-1}$ term $\left.\propto \cos \left(2 \omega_{0}\left(t^{\prime}+t^{\prime \prime}\right)\right)\right)$ we come to conclusion that at large $t$ only the term $\propto \cos \left(2 \omega_{0}\left(t^{\prime}-t^{\prime \prime}\right)\right)$ can give a remarkable contribution to the energy $E(t)$. Finally we take into account that at large time $t$ in the factor

$\cos \left(\omega_{k}\left(t^{\prime}-t^{\prime \prime}\right)\right) \cos \left(2 \omega_{0}\left(t^{\prime}-t^{\prime \prime}\right)\right)=\left(\cos \left(\left(\omega_{k}-2 \omega_{0}\right)\left(t^{\prime}-t^{\prime \prime}\right)\right)+\cos \left(\left(\omega_{k}+2 \omega_{0}\right)\left(t^{\prime}-t^{\prime \prime}\right)\right)\right) / 2$

only the term $\cos \left(\left(\omega_{0}-2 \omega_{0}\right)\left(t^{\prime}-t^{\prime \prime}\right)\right) / 2$ is essential. This gives

$$
E(t) \cong \frac{1}{8 N} \sum_{k} C_{k}^{2} \int_{0}^{t} d t^{\prime} \int_{0}^{t} d t^{\prime \prime} \cos \left(\left(\omega_{k}-2 \omega_{0}\right)\left(t^{\prime}-t^{\prime \prime}\right)\right)
$$

For small $\left|t^{\prime}-t^{\prime \prime}\right|$ being essential here one can replace the integral $\int_{0}^{t} \int_{0}^{t} d t^{\prime} d t^{\prime \prime}=$ $\int_{0}^{t} d t_{1} \int_{-t^{\prime}}^{t^{\prime}} d \tau$ by the integral $\int_{0}^{t} d t_{1} \int_{-\infty}^{\infty} d \tau$, where $t_{1}=\left(t^{\prime}+t^{\prime \prime}\right) / 2, \tau=t^{\prime}-t^{\prime \prime}$. As a result, in the $t \rightarrow \infty$ limit the rate of increasing of the energy of longitudinal phonons, followed by a subsequent decrease of the energy of the TILM under consideration, equals

$$
d E(t) / d t=(\pi / 4 N) \sum_{k} C_{k}^{2} \delta\left(\omega_{k}-2 \omega_{0}\right)
$$


Replacing here the sum by the integral and taking into account the relations $d m / d k=N / 2 \pi$ and $d k / d \omega_{k}=4 \omega_{k} / \sin k$ one finds

$$
d E / d t \approx\left(\omega_{0} \nu_{3}^{2} A_{0}^{4} / \sin k_{0}\right) \Phi^{2}(\Omega)
$$

where $\omega_{0}=\omega_{t m} \sqrt{1+\varepsilon^{2} / 4}, k_{0}=\arccos \left(1-8 \omega_{0}^{2} / \nu_{1}\right), \Omega=k_{0} / \varepsilon, \varepsilon=A_{0} \sqrt{3 \nu_{4}} / \omega_{t m}$

$$
\Phi(\Omega)=\int_{0}^{\infty} \frac{\sin (\Omega x) \sinh (x)}{\cosh ^{3}(x)} d x .
$$

The decay constant $\Gamma$ of the TILM is determined by the equation $\Gamma=E^{-1} d E / d t$. Taking into account the relation $E=\omega_{0}^{2} \sum_{n} y_{n}^{2}(0) / 2=\omega_{0}^{2} A_{0}^{2} / \varepsilon$, we get

$$
\Gamma=\frac{\varepsilon \nu_{3}^{2} A_{0}^{2}}{\omega_{0} \sin k_{0}} \Phi^{2}(\Omega) .
$$

$\Gamma$ decreases with decreasing of the amplitude of the central atom $A_{0}$ of the TILM. To get an estimation we consider the TILM with the amplitude $A_{0}=$ 0.03 in the $5 \%$ stretched chain $(s=0.05)$. In this chain $\omega_{t m} \approx 0.1880$, $\varepsilon \approx 0.183, \omega_{0} \approx 0.1888$, which gives $\Omega \approx 6$ and $\Phi^{2}(\Omega) \approx 2 \cdot 10^{-5}$. As a result we get $\Gamma \approx 5 \cdot 10^{-9}$. This corresponds to a very long lifetime.

\section{Transverse ILM in the chain; numerical study}

In our numerical study of transverse ILMs we considered a 5\% stretched chain with 40000 atoms and with fixed ends. The small compression of the entire chain due to TILM was taken into account. The potential energy was described by the interactions of the nearest neighbors with the Morse pair potential given by Eq. (1). We have found that TILMs indeed exist in this chain and they have a long lifetime (see Figs. 1-3; one period of the TILM with the frequency $\omega_{0} \approx 0.1888$ corresponds to 33 time units). E.g. in our numerical simulations we have found that the TILM with $A_{0}=0.03$ and $s=0.05$ has the frequency $\omega_{0} \approx 0.1883$ and the reversed size parameter $\varepsilon \approx 0.113$. It decays very slowly: its amplitude diminishes less than $2 \cdot 10^{-8}$ for 1000 periods of vibrations. This corresponds to $\Gamma<2 \cdot 10^{-11}$, which indeed gives very long lifetime, in agreement with the conclusion of the theory. 


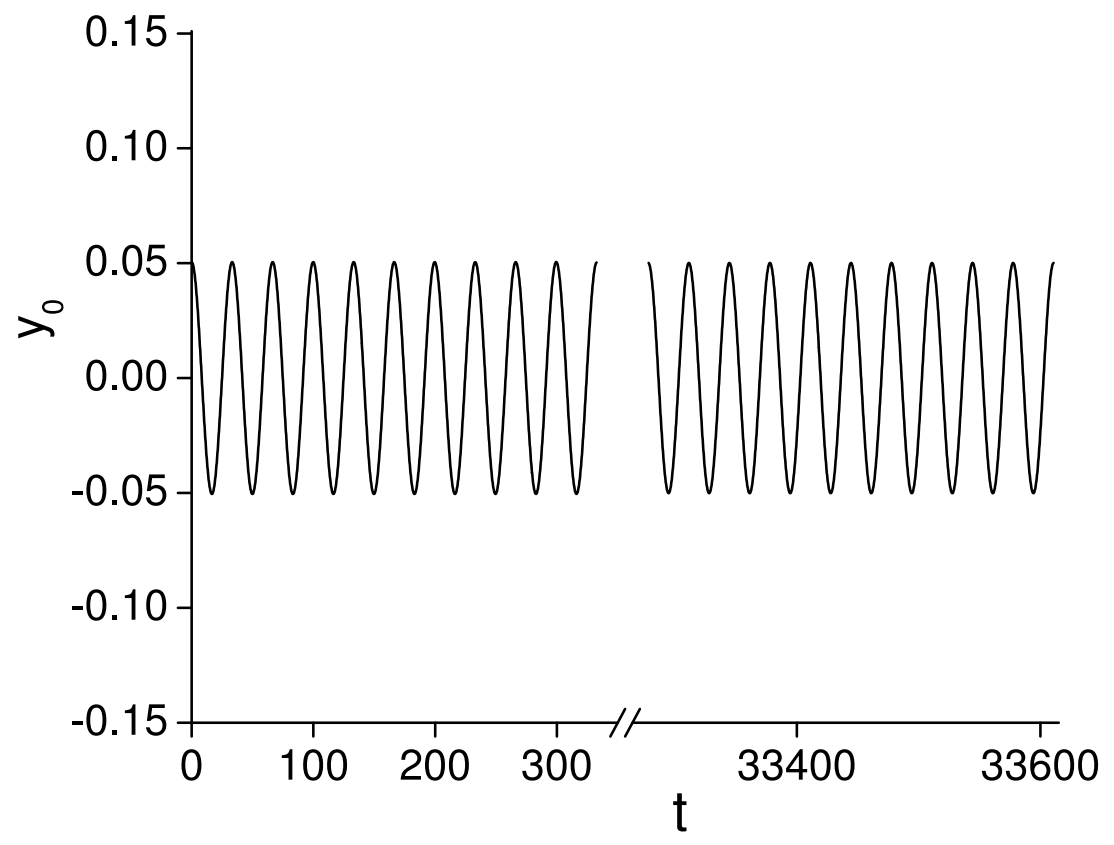

Fig. 1. Time dependence of the transverse shifts (in the units of atom spacing) of the central atom of a transverse IPLM in 5\% stretched monatomic chain; the initial shift of the central atom is 0.05 . First 10 periods and 10 periods after 1000 periods of vibration are presented.

The TILMs with the amplitudes $A_{0}>0.05$ decay faster; the rate of their decay rapidly increases with the increasing of the amplitude. This is also in agreement with the theory.

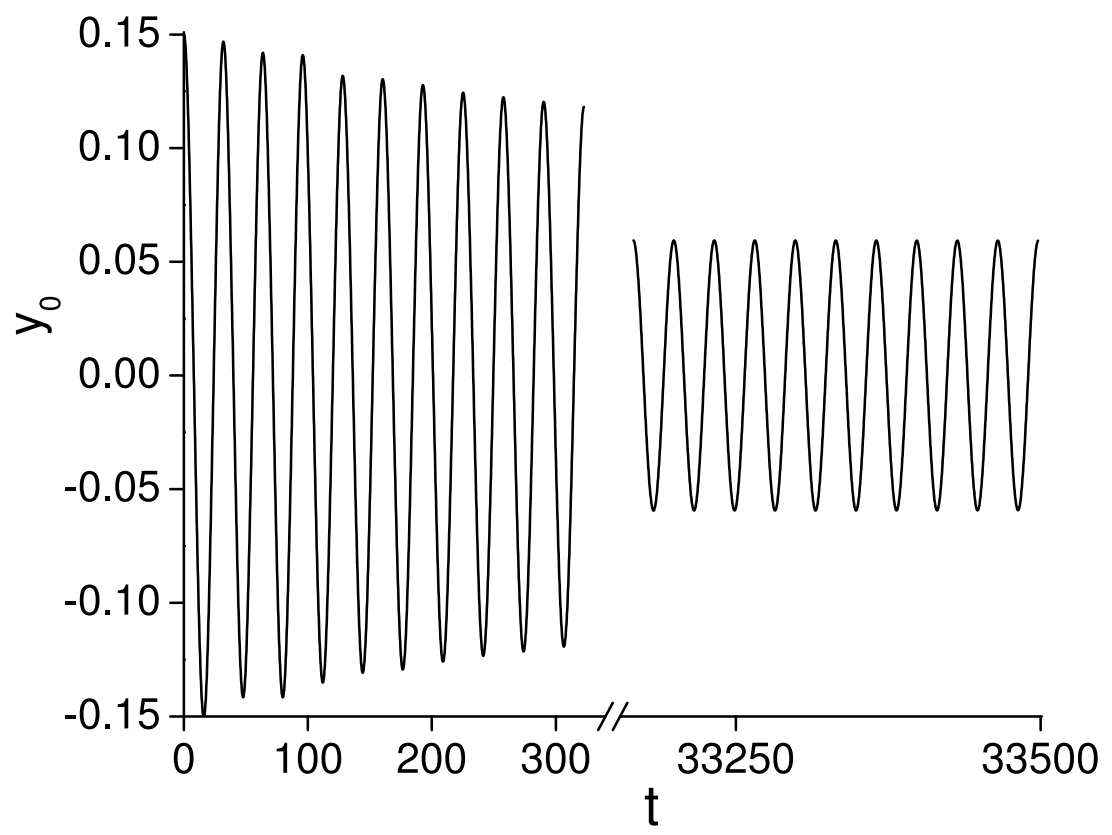

Fig. 2. The same as in Fig. 1, but the initial shift of the central atom of a transverse ILM is 0.15 . 


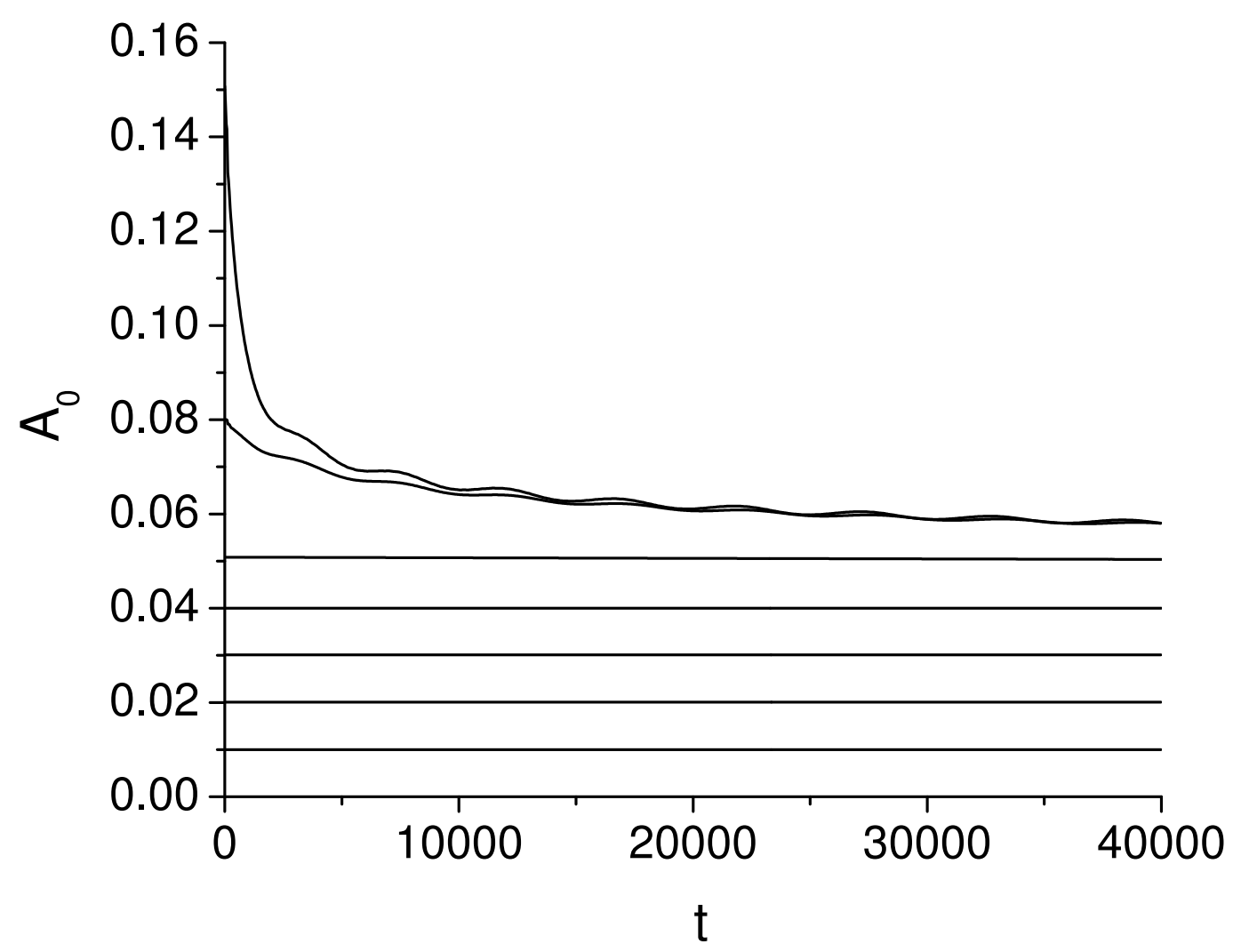

Fig. 3. Time dependence of the amplitudes of the central atom of transverse ILMs in $5 \%$ stretched monatomic chain for different initial shifts of the central atom.

Concerning the difference of the above-presented numerically-found values of the parameters $\varepsilon$ and $\omega_{0}$ from their theoretical values, we have found that these differences come from the neglecting of the local compression of the chain in the theory. This follows from the numerical simulations of TILMs in the chain rigid in $x$ direction (with $x$ coordinates of atoms being fixed at their values without TILM), performed by us. We have found the TILM in this chain with $\varepsilon \approx 0.183$ and $\omega_{0} \approx 0.1888$ in full agreement with the above theoretically found values of these parameters. This TILM does not decay, as it should according to the theory presented above. An approximate account of the compression mentioned in the theory, allowing one to explain the values $\varepsilon \approx 0.113$ and $\omega_{0} \approx 0.1883$ found in simulations, is given in Appendix. Note that if to use these values of $\varepsilon$ and $\omega_{0}$ in Eq. (21) we get $\Gamma \approx 2 \cdot 10^{-13}$. This also corresponds to a very long lifetime. 


\section{Graphene: out-of-plane vibrations}

The arguments presented in the beginning of Section 2 can readily be extended to planar atomic lattices. To be specific, we are considering here a graphene sheet. It consists of carbon atoms constituting the honeycomb-type periodical structure. Every carbon atom in this structure is connected with three neighboring atoms by chemical bonds formed by the $\mathrm{sp}_{2}$ hybrid orbitales.

We suppose that in equilibrium positions the graphene is situated in the $(x, y)$ plane. The distances between an atom and three neighboring atoms equal

$$
r_{n}=\sqrt{\left(b_{n} a+x_{n}\right)^{2}+\left(c_{n} a+y_{n}\right)^{2}+z_{n}^{2}}
$$

$(n=1,2,3)$, where $x_{n}, y_{n}$ and $z_{n}$ are the $x$-, $y$ - and $z$-components of the displacements of three neighboring atoms with respect to the central atom, $b_{1}=$ $0, b_{2}=\sqrt{3} / 2, b_{3}=-\sqrt{3} / 2, c_{1}=-1, c_{2}=1 / 2, c_{3}=1 / 2$. In the pair potential approximation the potential energy of the vibrations of atoms depends on the distances $R_{n}=r_{n}-a$ (explicit form of this energy in harmonic approximation, see e.g. in [16]). The expansion of these distances over the displacements $x_{n}, y_{n}$ and $z_{n}$ depends on the powers $R^{m}=\left(b x+c y+\left(x^{2}+y^{2}+z^{2}\right) / a\right)^{m}$ (here the subscript $n$ is omitted for simplicity). In the equilibrium position the $\propto R$ terms are cancelled. Therefore, the expansion of a pair potential over $z$ at $x=y=0$ starts with the positive quartic term $\propto z^{4}$, i.e. the $\propto z^{2}$ terms are absent. Consequently, in the pair potential approximation the $2 \mathrm{D}$ lattice is unstable with respect to small out-of-plane distortions. However, in graphene the atomic interactions are determined by covalent forces. These forces cause the stiffness of the planes with respect to the transverse short-range displacements of atoms [16]. In accordance with the Mermin-Wagner theorem [14] the plane of graphene remains unstable with respect to long-range transverse distortions, resulting in the appearance of ripples [17]18]. A stretching of the graphene sheet removes the ripples.

The elementary cell of graphene includes two atoms. Therefore, there are two branches of out-of-plane vibrations: acoustic and optic. One can expect the existence of two types of out-of-plane TILMs in graphene - with the frequency above the top frequency of acoustic out-of-plane phonons (the acoustic-like TILM) and with the frequency above the top frequency of optic out-of-plane phonons (the optic-like TILM). The acoustic-like out-of-plane TILMs can decay due to a relatively weak anharmonic interaction with the in-plane phonons and also due to in general stronger harmonic resonant interaction with outof-plane optic phonons. However, the optic-like out-of-plane TILMs can decay only due to a relatively weak anharmonic interaction with in-plane phonons. Therefore, the optic-type out-of-plane TILMs should have a longer lifetime. 
This conclusion is in agreement with our numerical modelling of out-of-plane TILMs in weakly-stretched $(1.5 \%)$ graphene. We have used the AIREBO potential and taken into account the cluster $246 \times 210 \AA(100 \times 50$ periods, 20 000 atoms) with periodical boundary conditions. Initially we displaced out-ofplane six atoms of $\mathrm{C}_{6}$ ring according to the optical mode. We found that outof-plane optic TILMs indeed exist; see Fig. 4 (where the time-dependences of the displacements of one of the three equivalent atoms of the central $\mathrm{C}_{6}$ ring are presented; the other three atoms in this ring vibrate out of phase with the mentioned atom) and Fig. 5 (where in-plane and out-of-plane phonons DOS and the spectrum of out-of-plane TILM for different initial amplitudes of carbon atoms are given).
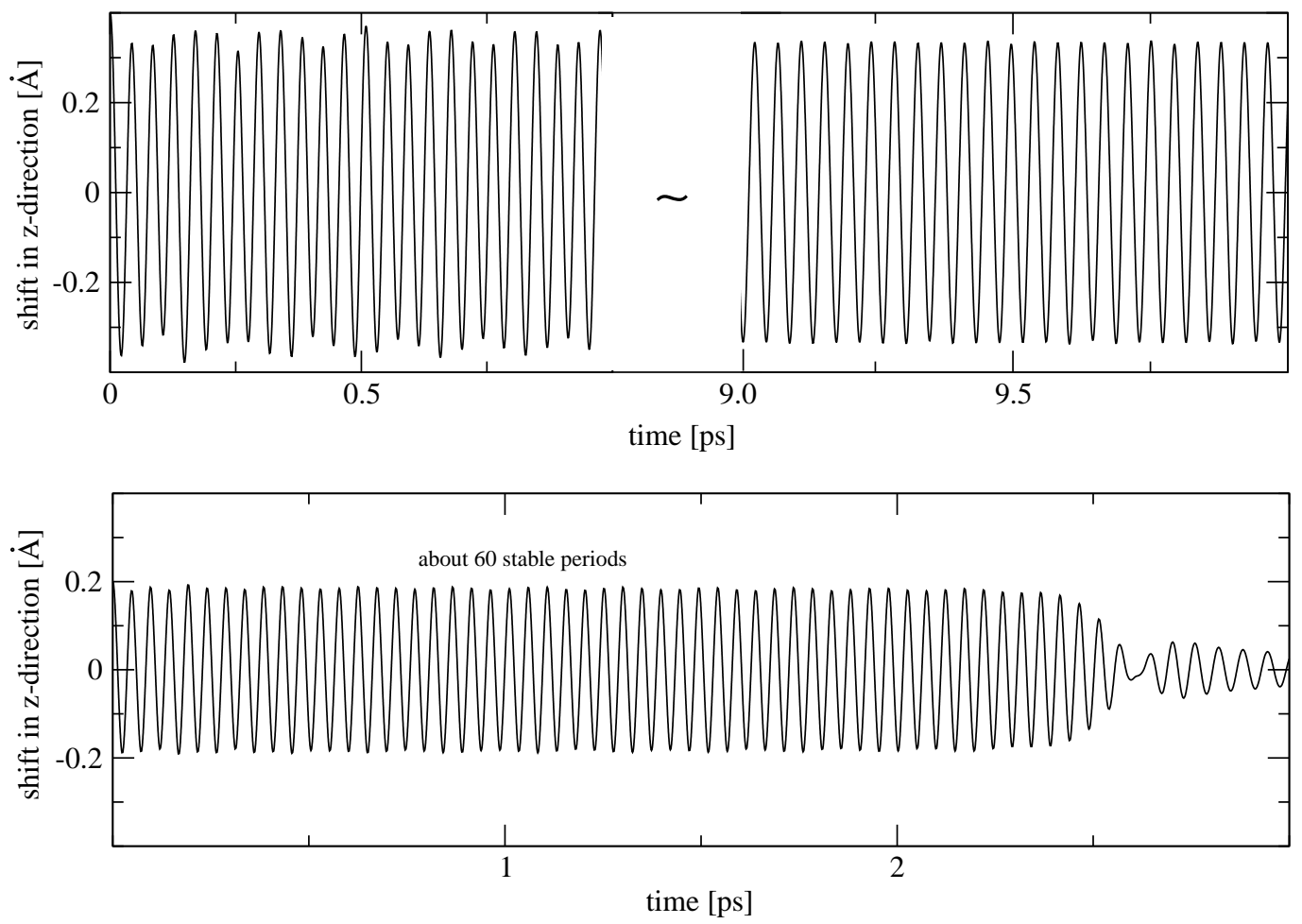

Fig. 4. Optical-type out-of-plane transverse ILMs in graphene. The out-of-plane shifts (independent of time) of one of three equivalent atoms of the central $\mathrm{C}_{6}$ ring is presented for initial amplitudes $0.4 \AA$ (upper) and $0.2 \AA$ (bottom); the other three atoms in this ring vibrate out of phase with the atom mentioned. 


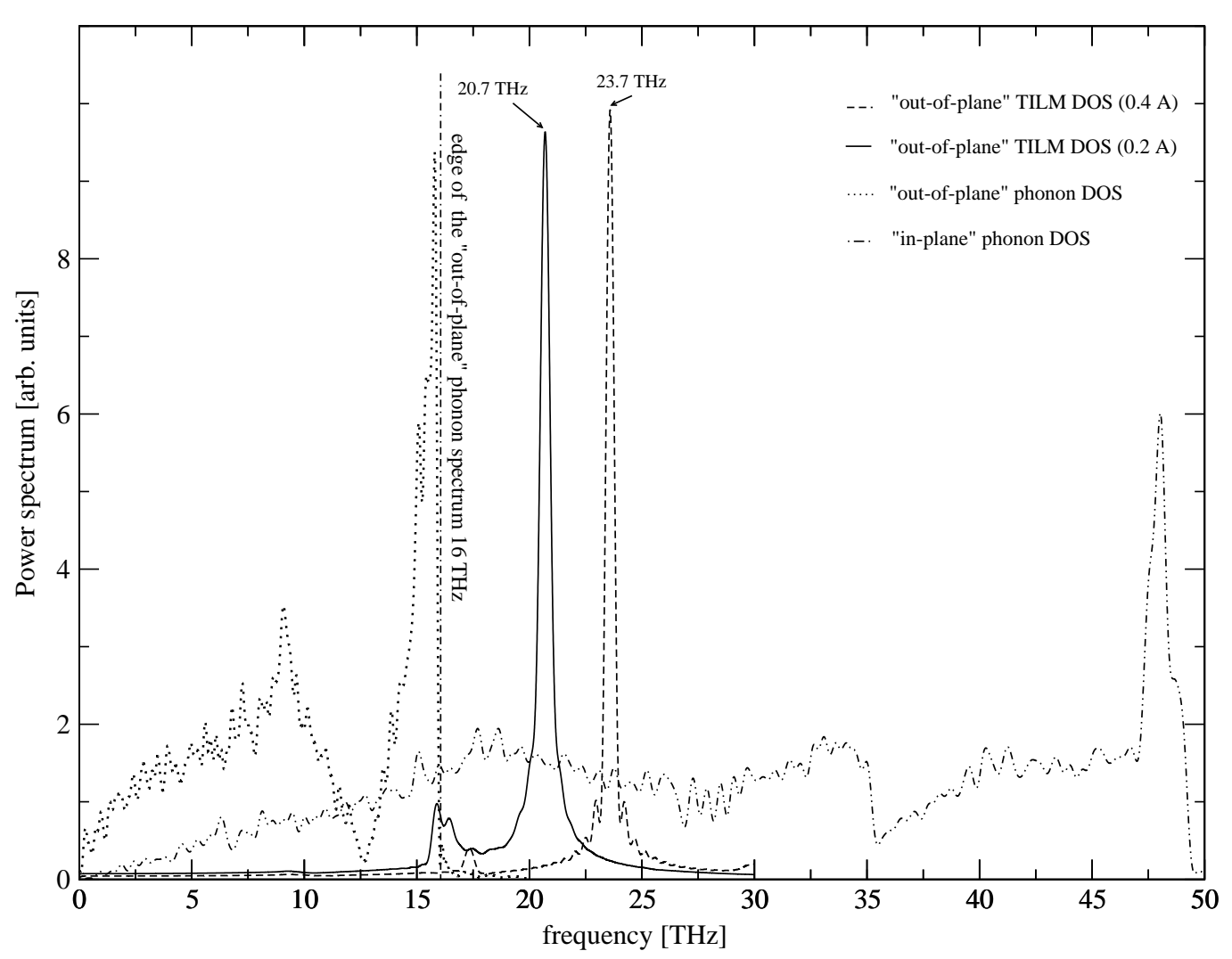

Fig. 5. In-plane and out-of-plane phonon DOS for the AIREBO potential and the spectrum of out-of-plane transverse ILM for different initial amplitudes of carbon atoms.

Note that in strongly-stretched graphene there can exist also in-plane anharmonic localized vibrations (in-plane discrete breathers) [19]20]. It appears (see Ref. [19]) that strong uniaxial stretching (along the zigzag or armchair direction) results in the opening of the gap in the middle of the phonon spectrum of graphene. This makes possible the existence of in-plane soft ILMs (discrete breathers) with the frequency in the gap of the phonon spectrum [19]20]. The authors [19,20] have found that these modes remain relatively stable also when, due to a large stretching, their frequencies get into resonance with out-of-plane phonons.

Thus, the physical situation discussed in Refs. [19|20] is reversed to the one considered here: the anharmonic in-plane modes with soft anharmonicity are in resonance with out-of-plane phonons, while out-of-plain TILMs considered here are in resonance with in-plane phonons. However, in both cases the lifetime of the modes is long. 


\section{Discussion}

In this communication it was shown that due to symmetry arguments in linear monatomic chains and in planar monatomic structures (e.g. graphene) odd anharmonicities disappear for the motion of atoms normal with respect to the chain/plane. However, even (quartic) anharmonicity exists and is hard (positive). As a result, the anharmonic localized modes of transverse (chain) or out-of-plane (graphene) vibrations (called TILMs) can exist in these systems with frequencies above the transverse/out-of-plane phonons. These vibrations have finite, although large lifetime: the slowly decay due to creation of longitudinal phonons. For the case of a monatomic chain a theory is being developed which allows one to calculate the decay rate of TILMs. The theory predicts that the lifetime of the TILMs under consideration may be extremely long, longer than $10^{10}$ periods of vibration. The numerical modelling of these TILMs fully confirms this result. The normal (out-of-plane) TILMs with the frequency above the phonon spectrum of out-of-plane phonons were also found for weakly-stretched graphene.

Here only immobile TILMs were considered. However, one can expect that these excitations can move in the same way as ILMs can move in $\mathrm{Cu}, \mathrm{Ni}$ and Fe in the nearest neighbors crystallographic directions [11,12,13].

The symmetry argument presented here holds generally for linear atomic chains and planar monolayer atomic structures. Therefore, one can expect the existence of TILMs in any linear atomic chain and any planar monolayer atomic structure. Besides, one can expect that an analogous situation may exist in 3D lattices with dominating chain and layer structural elements, e.g. in graphite and in mica. In such lattices odd anharmonicities exist, but they may be essentially reduced for out-of-chain/plane vibrations.

In this connection we would like to point to investigations of M. Russell et al. of muscovite mica irradiated by high energy particles (see, e.g. [21]). The irradiation produces in mica very long black tracks parallel to the crystallographic plains and localized between the layers. These tracks are explained in Ref. [21] by creation in the recoil process of discrete breathers which propagate on long distance along the crystallographic directions. The layered structure on atomic scale plays a crucial role in the observed phenomena. Although it is not yet clear whether these long-living vibrational excitations are transverse or longitudinal, the necessity of the layered structure on atomic scale to observe the phenomenon gives support to an assumption that these excitation should contain remarkable transverse component.

Analogous TILMs may be expected to exist also for surface vibrations in crystals with planar atomic surfaces. The change of the potential energy of 
a surface atom for the shift in outer direction also does not have any odd terms with respect to the shift. This leads to the decrease of the entire oddanharmonicity effects for out-of-plane surface vibrations, which may result in the appearance of out-of-plane TILMs with frequencies above the maximum frequency of transverse surface phonons and with a long lifetime.

It is known that next-to-next interactions in a chain can cause its zigzagtype secondary structure [22]. In such chains, e.g. in polyethylene, the mobile "solitons of tension" may exist [23] (involving the longitudinal-type motion of atoms). Besides, in such chains one should also expect the existence of transverse ILMs. Here two types of transverse vibrations should be distinguished: parallel and perpendicular to the plain of zigzag. The odd anharmonic terms for the transverse displacements perpendicular to the plain mentioned are absent; however, for transverse displacements parallel to this plain these are present. If the zigzag angle is large, then the TILMs perpendicular to the plain of zigzag should exist. However, if this angle is small, then the odd anharmonicity for the displacements parallel to the plain of zigzag is reduced. In such chains the existence of both TILMs is expected. It is worthwhile to note that intrinsic localized modes (discrete breathers) are often used to explain the targeted energy transfer in such basic for living organism organic chains as DNA [24,25] and proteins [26].

Acknowledgements. The research was supported by Estonian research projects SF0180013s07, IUT2-27 and by European Union through the European Regional Development Fund (project 3.2.01.11-0029).

\section{A The effect of self-compression on the shape of transverse ILM}

Let us consider the effect of longitudinal displacements of atoms, caused by the transverse ILM, to its shape and frequency. From Eq. (8) it follows that the TILM causes the change (reduction) of the atomic distances by $x_{c}=$ $-\nu_{3} y^{2}(0) / 4 \nu_{1}$ and slight vibrations of atoms in $x$ direction with the frequency $2 \omega_{0}$ (the forced vibrations). Neglecting the small vibrations mentioned, we find that on the average the $x$-dependent terms in Eq. (3) give the following mean (averaged over period of transverse vibrations) contribution to the potential energy:

$$
\bar{V}_{c}=\frac{\nu_{1}}{8} x_{c}^{2}+\frac{\nu_{3}}{8} x_{c} \overline{y^{2}}=\left(\frac{\nu_{3}^{2}}{128 \nu_{1}}-\frac{\nu_{3}^{2}}{64 \nu_{1}}\right) y^{4}(0)=-\frac{\nu_{3}^{2}}{128 \nu_{1}} y^{4}(0)
$$

Let us find the nonlinear interaction $V_{c}$ of a transverse motion with the mean value given by Eq. (A.1). We restrict ourselves to the nonlinear interaction of $\propto y^{4}$ type. Taking into account the relation $\overline{y^{4}}=3 y^{4}(0) / 8$, we get $V_{c}=$ $-\nu_{3}^{2} y^{4} / 48 \nu_{1}$. We suppose that this potential effectively accounts for the effect 
of displacements of atoms in $x$ direction. Adding this term to $V(0, y)$ in Eq. (5), we get the following effective potential energy of the transverse vibrations

$$
V_{e f f}\left(x_{c}, y\right)=V(0, y)+V_{c}=\frac{\nu_{2}}{8} s y^{2}+\frac{\tilde{\nu}_{4}}{32} y^{4}
$$

where $\tilde{\nu}_{4}=\nu_{4}-2 \nu_{3}^{2} / 3 \nu_{1}$. The TILM corresponding to this potential energy is described by Eq. (66) with the parameter

$$
\varepsilon=\sqrt{3 \tilde{\nu}_{4}} A_{0} / \omega_{t m}
$$

For the TILM with the amplitude $A_{0}=0.03$ of the central atom in $5 \%$ stretched chain $\tilde{\nu}_{4} \approx 0.167$. This gives $\varepsilon \approx 0.113$ and $\omega_{0} \approx 0.1883$ in agreement with numerically found values of $\varepsilon$ and $\omega_{0}$.

\section{References}

[1] A.A. Maradudin, E.W. Montroll, G.S. Weiss, I. P. Ipatova, Theory of lattice dynamics in the harmonic approximation, in: H. Ehrenreich, F. Seitz, D. Turnbull (Eds.), Solid State Physics, Suppl. 3, second ed., Academic Press, New York, 1971.

[2] A.M. Kosevich and A.S. Kovalev, Sov. Phys. JETP 40 (1974) 891.

[3] A.S. Dolgov, Sov. Phys. Solid State 28 (1986) 907.

[4] A.J. Sievers and S. Takeno, Phys. Rev. Lett. 61 (1988) 970.

[5] J.B. Page, Phys. Rev. B 41 (1990) 7835.

[6] A.J. Sievers and J.B. Page, in G.K. Norton and A.A. Maradudin (Eds.), Dynamical Properties of Solids: Phonon Physics The Cutting Edge, North Holland, Amsterdam, 1995, Vol. VII, p. 137.

[7] S. Flach and C.R. Willis, Phys. Repts. 295 (1998) 181.

[8] S.A. Kiselev and A.J. Sievers, Phys. Rev. B 55 (1997) 5755.

[9] M.Haas, V.Hizhnyakov, M.Klopov, A. Shelkan, IOP Conf. Series: Materials Science and Engineering, 15 (2010) 12045.

[10] N.K. Voulgarakis, S. Hadjisavvas, P.C. Kelires, and G.P. Tsironis, Phys. Rev. B 69 (2004) 113201.

[11] V.V. Hizhnyakov, M. Haas, A. Shelkan and M. Klopov, in: J.F.R. Archilla, N. Jiménez, V.J.Sánchez-Morcillo, L.M. García-Raffi (Eds.), Quodons in mica: nonlinear localized travelling excitations in crystals, Springer Series in Material Science, 221 (2015) 229. 
[12] M. Haas, V. Hizhnyakov, A. Shelkan, M. Klopov, A.J. Sievers, Phys. Rev. B 84 (2011) 144303.

[13] V. Hizhnyakov, M. Haas, A. Shelkan and M. Klopov, Phys. Scr. 89 (2014) 044003 .

[14] N.D. Mermin, Phys. Rev. 176 (1968) 250.

[15] S. Cadet, Phys. Lett. A 121 (1987) 77.

[16] V. Adamyan and V. Zavalniuk, J. Phys.: Condens. Matter 23 (2011) 015402.

[17] J.C. Meyer, A.K. Geim, M.I. Katsnelson, K.S. Novoselov, T.J. Booth and S. Roth, Nature 446 (2007) 60.

[18] A. Fasolino, J.H. Los and M.I. Katsenelson, Nature Materials 6 (2007) 858.

[19] L.Z. Khadeeva, S.V. Dmitriev, Yu.S. Kivshar, JETP Lett. 94 (2011) 539.

[20] J.A. Baimova, S.V. Dmitriev, K. Zhou, EPL 100 (2012) 36005.

[21] F.M. Russell and C.J. Eilbeck, Europhysics Letters, 78 (2007) 10004.

[22] A.V. Savin, L.I. Manevich, P.L. Christiansen and A.V. Zolotaryuk, Phys.-Usp. 42 (1999) 245.

[23] L.I. Manevitch and A.V. Savin, Phys. Rev. E, 55 (1997) 4713.

[24] M. Peyrard and A. R. Bishop, Phys. Rev. Lett., 62 (1989) 2755.

[25] P. Maniadis, B. S. Alexandrov, A. R. Bishop, and K. O. Rasmussen, Phys. Rev. E, 33 (2011) 011904.

[26] S. Luccioli, A. Imparato, S. Lepri, F. Piazza and A. Torcini, Phys. Biol., 8 (2011) 046008. 